\title{
$s$-wave scattering of a polarizable atom by an absorbing nanowire
}

\author{
Martin Fink, ${ }_{1}^{1}$ Andrés Naranjo, ${ }^{2}$ Florian Arnecke, ${ }^{1}$ Johannes Eiglsperger, ${ }^{1}$ Harald Friedrich, ${ }^{1,}$ Javier Madroñero, ${ }^{1}$ \\ Patrick Raab, ${ }^{1}$ and Andreas Wirzba ${ }^{3}$ \\ ${ }^{1}$ Physik Department, Technische Universität München, D-85747 Garching, Germany \\ ${ }^{2}$ Facultad de Ciencias, Universidad Nacional, Medellín, Colombia \\ ${ }^{3}$ Institute for Advanced Simulation and Institut für Kernphysik, Forschungszentrum Jülich, D-52425 Jülich, Germany
}

(Received 7 April 2010; published 28 June 2010)

\begin{abstract}
We study the scattering of a polarizable atom by a conducting cylindrical wire with incoming boundary conditions, that is, total absorption, near the surface of the wire. Based on the explicit expression given recently [C. Eberlein and R. Zietal, Phys. Rev. A 75, 032516 (2007)] for the nonretarded atom-wire potential, we formulate a hierarchy of approximations that enables the numerical determination of this potential to any desired accuracy as economically as possible. We calculate the complex $s$-wave scattering length for the effectively two-dimensional atom-wire scattering problem. The scattering length $\mathfrak{a}$ depends on the radius $R$ of the wire and a characteristic length $\beta$ related to the polarizability of the atom via a simple scaling relation, $\mathfrak{a}=R \tilde{\mathfrak{a}}(\beta / R)$. The "scaled scattering length" $\tilde{\mathfrak{a}}$ tends to unity in the thick-wire limit $\beta / R \rightarrow 0$, and it grows almost proportional to $1 / R$ in the opposite thin-wire limit.
\end{abstract}

DOI: 10.1103/PhysRevA.81.062714

PACS number(s): 34.50.-s, 03.65.Nk, 03.75.-b

\section{INTRODUCTION}

In view of the intense attention currently given to systems involving nanotubes at very low temperatures (see, e.g., [1]), it is important to understand fundamental processes involving the interaction of such cylindrical structures with cold atoms or molecules. The most basic process in this realm is the scattering of an atom by an infinite cylindrical conducting wire. Because of translational symmetry parallel to the cylinder axis, the system is effectively two-dimensional and it is separable in polar coordinates. Nevertheless, this seemingly simple problem is highly nontrivial for two reasons. (i) In contrast to effectively one-dimensional systems and to three-dimensional systems with radial symmetry, the interaction potential in realistic two-dimensional systems does not have a simple and well understood analytical structure. (ii) Scattering theory in two dimensions differs significantly from the well-studied three-dimensional case, in particular, in the low-energy, nearthreshold regime [2-4].

The interaction of a polarizable atom with an infinitely long and perfectly conducting cylinder has long been a subject of substantial interest (see, e.g., [5] and references therein). Practically useful expressions for the electrostatic van der Waals potential, which neglects retardation effects, have recently been given by Eberlein and Zietal [6]. With retardation included, the limits of large distance and of large conductivity are not interchangeable, so the model of a perfectly conducting wire loses its meaning at asymptotically large distances, as has been noted by Barash and Kyasov [7]. The problem can be treated formally, for example, by imposing the boundary conditions appropriate for a perfectly reflecting surface [8], but different results can be expected if the conductance properties of the wire are described via physically reasonable assumptions.

As a first step toward realistic calculations of low-energy atom-wire scattering, this paper presents exact numerical

\footnotetext{
*harald.friedrich@ph.tum.de
}

calculations of the $s$-wave scattering length for the nonretarded potential between a polarizable atom and a perfectly conducting cylinder. The radial Schrödinger equation is solved assuming incoming boundary conditions (total absorption) near the surface of the wire, which is realistic for the elastic scattering of fragile atoms such as metastable helium or alkali atoms. With total absorption near the surface of the wire, the scattering process corresponds to quantum reflection in the nonclassical region of the potential tail, and the complex scattering phase shifts as well as the complex scattering length do not depend on the details of the atom-wire interaction at very close distances, where it necessarily deviates from the singular van der Waals form.

In Sec. II we study the exact expression given recently in [6] for the nonretarded atom-wire potential. In a straightforward but nontrivial extension of a suggestion contained in [6], we formulate a hierarchy of approximations offering a practicable way of numerically calculating the nonretarded atom-wire potential with any desired accuracy as economically as possible. In Sec. III we calculate the $s$-wave scattering length for the effectively two-dimensional atom-wire system as a function of the radius $R$ of the wire, and we discuss the limits of large and small $R$.

\section{NONRETARDED ATOM-WIRE POTENTIAL}

Consider an atom at a distance $r$ from the axis of a perfectly conducting cylinder of radius $R$. The cylindrical coordinates of the atom are $r, \phi$, and $z$ (see Fig. 1). An exact expression for the electrostatic van der Waals potential $V(r)$ is given in Eq. (19) of Ref. [6]. In atomic units,

$$
\begin{aligned}
V(r)= & -\frac{1}{r^{3}}\left[\left\langle\hat{d}_{r}^{2}\right\rangle X_{r}\left(\frac{r}{R}\right)+\left\langle\hat{d}_{\phi}^{2}\right\rangle X_{\phi}\left(\frac{r}{R}\right)\right. \\
& \left.+\left\langle\hat{d}_{z}^{2}\right\rangle X_{z}\left(\frac{r}{R}\right)\right] .
\end{aligned}
$$

The angle brackets represent the expectation values of the squares of the cylindrical components of the dipole operator $\hat{\vec{d}}$. For a spherical atomic state, the three expectation values 


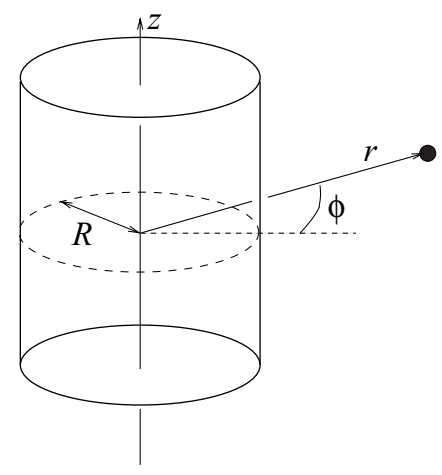

FIG. 1. Schematic illustration of the atom-wire system in cylindrical coordinates.

are equal, $\left\langle\hat{d}_{r}^{2}\right\rangle=\left\langle\hat{d}_{\phi}^{2}\right\rangle=\left\langle\hat{d}_{z}^{2}\right\rangle$. The dimensionless coefficients $X_{r}, X_{\phi}, X_{z}$ are functions of the ratio $\rho=r / R$ of the radial distance $r$ and the cylinder radius $R$, and they are given as sums of integrals involving the modified Bessel functions $I_{m}$ and $K_{m}$ [9],

$$
\begin{aligned}
& X_{r}(\rho)=\frac{1}{\pi} \sum_{m=-\infty}^{+\infty} \int_{0}^{\infty} \frac{I_{m}(\eta / \rho)}{K_{m}(\eta / \rho)}\left[K_{m}^{\prime}(\eta)\right]^{2} \eta^{2} d \eta, \\
& X_{\phi}(\rho)=\frac{1}{\pi} \sum_{m=-\infty}^{+\infty} m^{2} \int_{0}^{\infty} \frac{I_{m}(\eta / \rho)}{K_{m}(\eta / \rho)}\left[K_{m}(\eta)\right]^{2} d \eta, \\
& X_{z}(\rho)=\frac{1}{\pi} \sum_{m=-\infty}^{+\infty} \int_{0}^{\infty} \frac{I_{m}(\eta / \rho)}{K_{m}(\eta / \rho)}\left[K_{m}(\eta)\right]^{2} \eta^{2} d \eta ;
\end{aligned}
$$

they are related to the functions $\Xi_{j}$ of [6] by

$$
X_{j}\left(\frac{r}{R}\right)=r^{3} \Xi_{j}(r, R), \quad j=r, \phi, z .
$$

The atom-wire potential thus has the general form

$$
V(r) \propto-\frac{1}{r^{3}} X\left(\frac{r}{R}\right) .
$$

For a spherical atom, we have

$$
V(r)=-\frac{\left\langle\psi\left|\hat{d}_{j}^{2}\right| \psi\right\rangle}{r^{3}} X\left(\frac{r}{R}\right),
$$

where $j$ stands for any one of the cylindrical coordinates, $r$, $\phi$, or $z$. The expectation value $\left\langle\psi\left|\hat{d}_{j}^{2}\right| \psi\right\rangle$ in (7) depends only on the polarization properties of the atom (or molecule), and the factor $X$ is a universal function of $\rho=r / R$, independent of which atom (or molecule) we are considering:

$$
X(\rho)=X_{r}(\rho)+X_{\phi}(\rho)+X_{z}(\rho) .
$$

As the radial distance approaches the cylinder radius, $\rho \rightarrow 1$, the factor $X$ behaves as [6],

$$
X(\rho) \stackrel{\rho \rightarrow 1}{\sim} \frac{\rho^{3}}{4(\rho-1)^{3}},
$$

so we obtain the well-known result $[10,11]$ for the nonretarded van der Waals potential of an atom at the distance $r-R$ from a plane conducting surface,

$$
V(r) \stackrel{r \rightarrow R}{\sim}-\frac{C_{3}}{(r-R)^{3}}, \quad C_{3}=\frac{1}{4}\left\langle\psi\left|\hat{d}_{j}^{2}\right| \psi\right\rangle .
$$

From the low-argument behavior of the modified Bessel functions, it follows that the terms with $m \neq 0$ on the righthand sides of Eqs. (2)-(4) behave as $1 / \rho^{2|m|}$ asymptotically. The leading contributions to $X(\rho)$ come from the $m=0$ terms in the sums in Eqs. (2) and (4), which are proportional to $1 / \ln (\rho)$ in the limit of large $\rho$. This information is not very helpful, however, as the next terms differ only through higher terms in $1 / \ln (\rho)$, and such logarithmic series converge very slowly [6]. Furthermore, neglecting the terms with $m \neq 0$ is a poor approximation except for very large values of $\rho$ [6]. An improvement suggested in [6] involves explicitly retaining the $m=0$ terms in Eqs. (2) and (4) and treating the residual sums with the help of the uniform large-order approximation for the Bessel fuctions [9]. Here we generalize this ansatz by allowing an arbitrary finite number of terms to be retained in the sums in Eqs. (2)-(4) and only applying the large-order approximation to the residual sum containing the terms with $|m|>m_{\max }$.

The residual-sum contribution to $X_{z}$ is

$$
X_{z}^{m_{\max }}(\rho)=\frac{2}{\pi} \sum_{m=\nu}^{\infty} \int_{0}^{\infty} \frac{I_{m}(\eta / \rho)}{K_{m}(\eta / \rho)}\left[K_{m}(\eta)\right]^{2} \eta^{2} d \eta,
$$

where $v=m_{\max }+1$. Changing the integration variable to $x=\eta / m$ gives

$$
X_{z}^{m_{\max }}(\rho)=\frac{2}{\pi} \sum_{m=v}^{\infty} \int_{0}^{\infty} m^{3} \frac{I_{m}(m x / \rho)}{K_{m}(m x / \rho)}\left[K_{m}(m x)\right]^{2} x^{2} d x .
$$

Using the uniform large-order approximations for the Bessel functions [9],

$$
\begin{gathered}
I_{m}(m z) \approx \frac{1}{\sqrt{2 \pi m}} \frac{e^{m \zeta}}{\left(1+z^{2}\right)^{1 / 4}}, \\
K_{m}(m z) \approx \sqrt{\frac{\pi}{2 m}} \frac{e^{-m \zeta}}{\left(1+z^{2}\right)^{1 / 4}}, \\
K_{m}^{\prime}(m z) \approx-\sqrt{\frac{\pi}{2 m}} \frac{e^{-m \zeta}}{z}\left(1+z^{2}\right)^{1 / 4}, \\
\zeta=\sqrt{1+z^{2}}+\ln \left(\frac{z}{1+\sqrt{1+z^{2}}}\right),
\end{gathered}
$$

allows explicit execution of the summation, so

$$
X_{z}^{m_{\max }}(\rho)=\frac{1}{\pi} \int_{0}^{\infty} \frac{\xi^{2}}{\sqrt{1+\xi^{2}}} J(\nu, \xi, \rho) d \xi .
$$

The function $J(v, \xi, \rho)$ is given by

$$
J(v, \xi, \rho)=\frac{A^{v}\left(A^{2}(v-1)^{2}+A\left(1+2 v-2 v^{2}\right)+v^{2}\right)}{(1-A)^{3}},
$$

where $A$ is a function of the integration variable $\xi$ and of $\rho=r / R$,

$$
A=\frac{e^{-2\left(\sqrt{1+\xi^{2}}-\sqrt{1+\xi^{2} / \rho^{2}}\right)}}{\rho^{2}}\left(\frac{1+\sqrt{1+\xi^{2}}}{1+\sqrt{1+\xi^{2} / \rho^{2}}}\right)^{2} .
$$


Similar operations yield the residual-sum contributions to $X_{r}$ and $X_{\phi}$ :

$$
\begin{aligned}
& X_{r}^{m_{\max }}(\rho)=\frac{1}{\pi} \int_{0}^{\infty} \sqrt{1+\xi^{2}} J(v, \xi, \rho) d \xi \\
& X_{\phi}^{m_{\max }}(\rho)=\frac{1}{\pi} \int_{0}^{\infty} \frac{1}{\sqrt{1+\xi^{2}}} J(\nu, \xi, \rho) d \xi
\end{aligned}
$$

We have thus defined a hierarchy of approximations to the exact atom-wire potential with levels characterized by the summation index $m_{\max }$ up to which the terms in the sums in Eqs. (2), (3), and (4) are included exactly,

$$
\begin{aligned}
X_{r}(\rho)= & \frac{1}{\pi} \sum_{m=-m_{\max }}^{+m_{\max }} \int_{0}^{\infty} \frac{I_{m}(\eta / \rho)}{K_{m}(\eta / \rho)} \\
& \times\left[K_{m}^{\prime}(\eta)\right]^{2} \eta^{2} d \eta+X_{r}^{m_{\max }}(\rho), \\
X_{\phi}(\rho)= & \frac{1}{\pi} \sum_{m=-m_{\max }}^{+m_{\max }} m^{2} \int_{0}^{\infty} \frac{I_{m}(\eta / \rho)}{K_{m}(\eta / \rho)} \\
& \times\left[K_{m}(\eta)\right]^{2} d \eta+X_{\phi}^{m_{\max }}(\rho), \\
X_{z}(\rho)= & \frac{1}{\pi} \sum_{m=-m_{\max }}^{+m_{\max }} \int_{0}^{\infty} \frac{I_{m}(\eta / \rho)}{K_{m}(\eta / \rho)} \\
& \times\left[K_{m}(\eta)\right]^{2} \eta^{2} d \eta+X_{z}^{m_{\max }}(\rho) ;
\end{aligned}
$$

the residual-sum contributions $X_{j}^{m_{\max }}(\rho)$ are as given in Eqs. (14), (17), and (18). In this approximation, the large- $\rho$ behavior of the nonretarded atom-wire potential (7) is treated exactly up to and including terms of order $1 / \rho^{4+2 m_{\max }}$. This straightforward but nontrivial generalization of the approximation suggested in [6] offers a practicable way of numerically calculating the nonretarded atom-wire potential with any desired accuracy as economically as possible.

Numerical results obtained in various approximations for $X(\rho)$ [Eq. (8)] are shown in Fig. 2. For $m_{\max }=5$, the potential

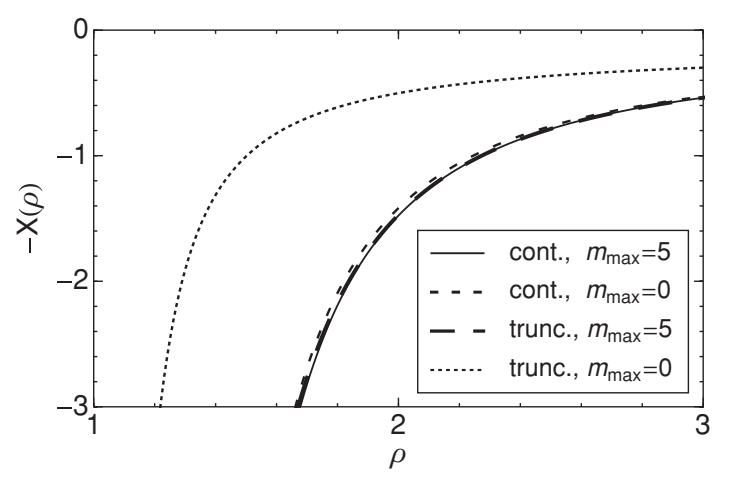

FIG. 2. Numerical calculation of the universal function (8). The solid line shows the result of the approximation (19), where the contributions in Eqs. (2)-(4) are included exactly for $|m| \leqslant m_{\max }=5$, while the sums are continued to higher $|m|$ via the uniform largeorder approximation of the Bessel functions according to (14), (17), and (18). The short-dashed line shows the corresponding result for $m_{\max }=0$. The dotted line shows the result obtained when expansions (2)-(4) are truncated after $m_{\max }=0$ and the contributions from higher $|m|$ are neglected altogether; the long-dashed line is the result of the corresponding truncated expansion with $m_{\max }=5$.

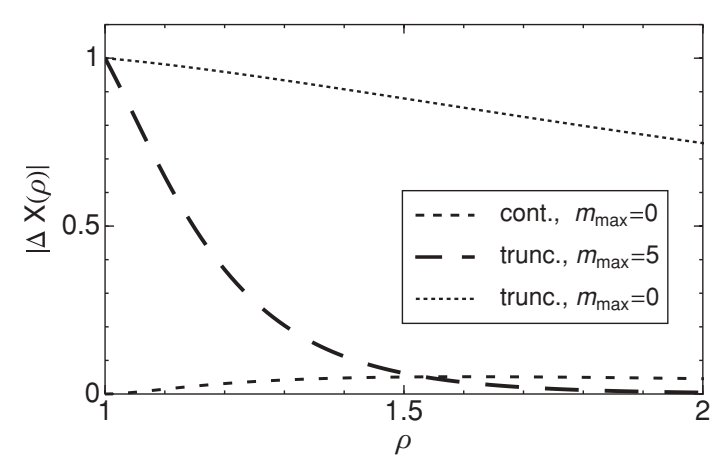

FIG. 3. Absolute value of the relative error (20) of various approximations to the universal function (8). The short-dashed line is the result of approximation (19), when only the $m=0$ contributions in Eqs. (2) and (4) are included exactly, while the sums are continued to higher $|m|$ via the uniform large-order approximation of the Bessel functions according to (14), (17), and (18). The dotted line shows the result obtained by truncating the sums after $m=0$ and neglecting the contributions from $|m|>0$. The long-dashed line corresponds to truncation after $m_{\max }=5$.

defined by (19) (solid line in Fig. 2) has converged to a relative accuracy better than $1 \%$ in the whole range $1<\rho<\infty$, and it is essentially indistinguishable from the exact result. As noted in [6], calculating only the $m=0$ contributions exactly and continuing the sum over higher $|m|$ via the large-order approximation of the Bessel functions, that is, taking $m_{\max }=0$ in (19), approximates the exact potential quite well, as shown by the short-dashed line in Fig. 2. Truncating the sums in Eqs. (2)-(4) after $m=0$ without including the contributions from higher $|m|$ even approximately (dotted line in Fig. 2) clearly gives a very poor approximation. Truncating the sums in Eqs. (2)-(4) after a higher $m_{\max }$, for example, $m_{\max }=5$ (long-dashed line in Fig. 2), seems to give better results, but this is only true for large $\rho$. To illustrate this point, Fig. 3 shows the relative error,

$$
\Delta X(\rho) \stackrel{\text { def }}{=} \frac{X^{\mathrm{appr}}(\rho)-X(\rho)}{X(\rho)},
$$

of various approximations $X^{\text {appr }}(\rho)$ to $X(\rho)$. For the approximation based on $m_{\max }=0$ in (19) (short-dashed line), the relative error remains of the order of a few percent in the whole range $1<\rho<\infty$, whereas the "truncated approximation" neglecting the contributions from higher $|m|$ gives a relative error of order unity (dotted line in Fig. 3). Even when the contributions are included exactly up to a higher value of $m_{\max }$, the truncated approximation (neglecting contributions $|m|>m_{\max }$ ) remains poor, except for high $\rho$. This is illustrated for the case $m_{\max }=5$ in Fig. 3. The truncated approximation with $m_{\max }=5$ (long-dashed line) is better than the truncated approximation with $m_{\max }=0$ (dotted line), but the relative error still approaches unity for $\rho \rightarrow 1$. This shows that the singular behavior (9) of $X(\rho)$ for $\rho \rightarrow 1$ is not reproduced correctly when the sums in Eqs. (2)-(4) are truncated at a finite $m_{\max }$. An approximate treatment of the higher terms $|m|>m_{\max }$ according to (14), (17), and (18) restores the correct behavior (9) for $\rho \rightarrow 1$. 


\section{ATOM-WIRE SCATTERING}

Due to translational symmetry in the direction parallel to the axis of the wire, the problem of atom-wire scattering effectively reduces to the two-dimensional problem of an atom moving in the plane perpendicular to the symmetry axis of the wire. Such a two-dimensional scattering problem is significantly different from the well-studied three-dimensional case, in particular, in the low-energy, near-threshold regime $[3,12]$.

\section{A. Scattering by a radially symmetric potential in two dimensions}

Two-dimensional scattering theory has been receiving considerable attention recently [12-14]. The essential differences to the three-dimensional case were already formulated in the 1980s [2-4]. The partial-wave expansions of the wave function $\psi(\vec{r})$ and the scattering amplitude $f(\phi)$ read

$$
\psi(\vec{r})=\sum_{\mu=-\infty}^{+\infty} \frac{u_{\mu}(r)}{\sqrt{r}} e^{i \mu \phi}, \quad f(\phi)=\sum_{\mu=-\infty}^{+\infty} f_{\mu} e^{i \mu \phi} .
$$

The radial wave functions $u_{\mu}(r)$ solve the radial Schrödinger equation,

$$
\frac{d^{2} u_{\mu}}{d r^{2}}+\left[k^{2}-\frac{\mu^{2}-1 / 4}{r^{2}}-\frac{2 M}{\hbar^{2}} V(r)\right] u_{\mu}(r)=0,
$$

and behave asymptotically as

$$
u_{\mu}(r) \stackrel{r \rightarrow \infty}{\propto} \sin \left(k r-|\mu| \frac{\pi}{2}+\frac{\pi}{4}+\delta_{\mu}\right) .
$$

The partial wave amplitudes $f_{\mu}$ in (21) are related to the scattering phase shifts $\delta_{\mu}$ in (23) via (see, e.g., [12])

$$
f_{\mu}=\frac{1}{\sqrt{2 i \pi k}}\left(e^{2 i \delta_{\mu}}-1\right) .
$$

The two-dimensional centrifugal potential in (22) differs from the familiar three-dimensional expression in that it contains $\mu^{2}-\frac{1}{4}$ in place of $l(l+1)$. This means that $|\mu|$ corresponds to $l+\frac{1}{2}$. For sufficiently short-ranged potentials, Wigner's threshold law for phase shifts thus reads

$$
\tan \delta_{\mu} \stackrel{k \rightarrow 0}{\propto} k^{2|\mu|}, \quad \mu \neq 0 .
$$

Toward threshold, the scattering amplitude is dominated by the $s$ wave $(\mu=0)$, for which the near-threshold behavior of the phase shift is given by

$$
\cot \delta_{0} \stackrel{k \rightarrow 0}{\sim} \frac{2}{\pi}\left[\ln \left(\frac{k a}{2}\right)+\gamma_{\mathrm{E}}\right]
$$

$\gamma_{\mathrm{E}}$ is Euler's constant, $\gamma_{\mathrm{E}}=0.577 \ldots$ The quantity $a$ in (26) is the two-dimensional version of the scattering length [3,12-14], and it is well defined for potentials falling off faster than $1 / r^{2}$ at large distances [12]. The asymptotic form of the $s$-wave radial wave function at threshold $(k=0)$ is

$$
u_{0}^{(0)}(r) \stackrel{r \rightarrow \infty}{\propto}-\sqrt{r} \ln \left(\frac{r}{a}\right) .
$$

Toward threshold, where the scattering amplitude is dominated by $s$ waves,

$$
\begin{aligned}
f(\phi) & \equiv f_{0}=\frac{1}{\sqrt{2 \pi i k}}\left(e^{2 i \delta_{0}}-1\right)=\frac{\sqrt{2 i /(\pi k)}}{\cot \delta_{0}-i} \\
& \stackrel{\sqrt{\pi i /(2 k)}}{\sim} \frac{\ln (k a / 2)+\gamma_{\mathrm{E}}-i \frac{\pi}{2}}{\ln } .
\end{aligned}
$$

For potentials which are attractive and more singular than $-1 /(r-R)^{2}$ toward the surface of the cylinder, the semiclassical WKB approximation becomes increasingly accurate as $r$ approaches $R$, and it can be used to define incoming boundary conditions in this region. This implies total absorption at the surface of the wire, and all flux observed in elastic scattering is due to quantum (or classical) reflection in the potential tail. The scattering phase shifts are now complex. At threshold, the $\mu=0$ radial wave function $u_{0}^{\text {inc }}(r)$, which obeys incoming boundary conditions for $r \rightarrow R$, behaves asymptotically as

$u_{0}^{\text {inc }}(r) \stackrel{r \rightarrow \infty}{\propto}-\sqrt{r} \ln \left(\frac{r}{\mathfrak{a}}\right)=-\sqrt{r} \ln \left(\frac{r}{|\mathfrak{a}|}\right)+i \arg (\mathfrak{a}) \sqrt{r}$

$\mathfrak{a}$ is the $s$-wave scattering length, which is now also complex. The modulus and phase of $\mathfrak{a}$ dominantly determine the nearthreshold behavior of the scattering amplitude,

$$
f(\phi) \stackrel{k \rightarrow 0}{\sim} \frac{\sqrt{\pi i /(2 k)}}{\ln (k|\mathfrak{a}| / 2)+\gamma_{\mathrm{E}}+i\left(\arg (\mathfrak{a})-\frac{\pi}{2}\right)},
$$

and of the differential scattering cross section,

$$
\begin{aligned}
\frac{d \lambda}{d \phi}=|f(\phi)|^{2} \stackrel{k \rightarrow 0}{\sim} & \frac{\pi /(2 k)}{\left[\ln (k|\mathfrak{a}| / 2)+\gamma_{\mathrm{E}}\right]^{2}+\left[\arg (\mathfrak{a})-\frac{\pi}{2}\right]^{2}} \\
& +O\left(k^{0}\right) .
\end{aligned}
$$

Note that both the modulus and the phase of the complex scattering length $\mathfrak{a}$ are uniquely defined via the asymptotic behavior (29) of $u_{0}^{\text {inc }}$, and there is no ambiguity in the phase.

In three-dimensional scattering or quantum reflection, the $s$-wave scattering length determines the near-threshold behavior of the differential scattering cross section very directly according to $|f(\theta)|^{2} \stackrel{k \rightarrow 0}{\sim}|\mathfrak{a}|^{2}[1+2 k \operatorname{Im}(\mathfrak{a})][15]$. In the two-dimensional case (31), the differential cross section diverges essentially as $1 / k$ for $k \rightarrow 0$. The denominator containing the squared logarithmic term is an important factor for determining the absolute value of the cross section for $k|\mathfrak{a}| \ll 2$, and it slightly alleviates the $1 / k$ divergence in this regime.

\section{B. Application to atom-wire scattering}

Inserting the nonretarded potential (7) for a spherical atom and a wire of radius $R$ into the radial Schrödinger equation (22), and assuming $s$ waves $(\mu=0)$ as well as vanishing energy $(k=0)$, leads to

$$
\frac{d^{2} u_{0}}{d r^{2}}+\left[\frac{1 / 4}{r^{2}}+\frac{\beta}{r^{3}} X\left(\frac{r}{R}\right)\right] u_{0}(r)=0 ;
$$


TABLE I. Expectation value of a dipole component squared and the associated characteristic length $\beta$ as defined in (33) for various atoms - in their ground state unless otherwise specified.

\begin{tabular}{lccccccccc}
\hline \hline & $\mathrm{H}^{\mathrm{a}}$ & $\mathrm{He}^{\mathrm{b}}$ & $\mathrm{He}\left(2^{1} S\right)^{\mathrm{c}}$ & $\mathrm{He}\left(2^{3} S\right)^{\mathrm{c}}$ & $\mathrm{Li}^{\mathrm{d}}$ & $\mathrm{Na}^{\mathrm{e}}$ & $\mathrm{K}^{\mathrm{f}}$ & $\mathrm{Rb}^{\mathrm{f}}$ & $\mathrm{Cs}^{\mathrm{f}}$ \\
\hline$\left\langle\psi\left|\hat{d}_{j}^{2}\right| \psi\right\rangle$ (a.u.) & 1 & 0.752 & 10.68 & 7.60 & 6.07 & 7.56 & 11.44 & 13.44 & 16.56 \\
$\beta / 10^{3}$ (a.u.) & 3.67 & 11.0 & 156 & 111 & 154 & 634 & 1630 & 4190 & 8020 \\
\hline \hline
\end{tabular}

${ }^{\text {aFrom Ref. [16]. }}$

${ }^{\mathrm{b}}$ From Ref. [17].

${ }^{c}$ From Ref. [18].

${ }^{\mathrm{d}}$ From Ref. [19].

${ }^{\mathrm{e}}$ From Ref. [20].

${ }^{\mathrm{f}}$ From Ref. [21].

here $X$ is the universal function defined in (8), and $\beta$ is a characteristic length related to the polarizability of the atom,

$$
\beta=\frac{2 M}{\hbar^{2}}\left\langle\psi\left|\hat{d}_{j}^{2}\right| \psi\right\rangle .
$$

The characteristic length $\beta$ is related to the coefficient $C_{3}$ of the $1 /(r-R)^{3}$ behavior of the potential near the surface (10) by

$$
\frac{\beta}{4}=\frac{2 M}{\hbar^{2}} C_{3} \text {. }
$$

Since the matrix element in (33) is usually of the order of a few atomic units, the length $\beta$ is generally quite large, because the mass $M$ of the atom is large (in atomic units). Table I lists, as examples, explicit values for hydrogen and several alkali atoms in their respective ground state and helium in its ground or excited metastable $2 S$ state. Values of $\beta$ range from a few thousand to several million Bohr radii. A radius $R$ of the nanowire between 20 and $500 \mathrm{~nm}$, say, would correspond to a ratio $R / \beta$ roughly between 0.1 and 2.5 for the hydrogen atom and between $5 \times 10^{-5}$ and $1.2 \times 10^{-3}$ for cesium.

The solution $u_{0}^{\text {inc }}$ of (32) which obeys incoming boundary conditions for $r \rightarrow R$ behaves asymptotically according to (29), and this defines the complex scattering length $\mathfrak{a}$, which dominantly determines the atom-wire scattering process at near-threshold energies according to (30) and (31).

Figure 4 shows the absolute value of the scattering length obtained by solving the radial Schrödinger equation (32). The solid line shows the essentially exact result based on the potential (19), where the contributions in Eqs. (2)-(4) are included exactly for $|m| \leqslant m_{\max }=5$, while the sums are continued to higher $|m|$ via the uniform large-order approximation of the Bessel functions according to (14), (17), and (18). The short-dashed line shows the corresponding result obtained for $m_{\max }=0$. There is a small but noticeable deviation from the exact result. The long-dashed line in Fig. 4 shows the result obtained when the expansions (2)-(4) are truncated after $m_{\max }=5$ and the contributions from higher $|m|$ are neglected altogether. Although the asymptotic behavior of the potential is now reproduced exactly up to terms of order $1 / \rho^{14}$, it gives a very poor result for the scattering length. As discussed in connection with Fig. 3, truncating the sums after a finite $m_{\max }$ without appropriate consideration of the contributions from higher $|m|$ leads to a dramatically wrong behavior of $X(\rho)$ for $\rho \rightarrow 1$, and this obviously has devastating effects on the result obtained for the scattering length.

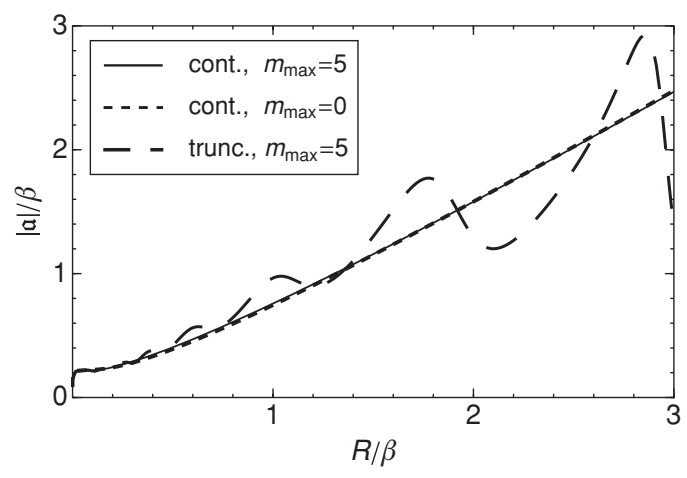

FIG. 4. Absolute value $|\mathfrak{a}|$ of the $s$-wave scattering length. The solid line shows the essentially exact result based on potential (19), where the contributions in Eqs. (2)-(4) are included exactly for $|m| \leqslant m_{\max }=5$, while the sums are continued to higher $|m|$ via the uniform large-order approximation of the Bessel functions according to (14), (17), and (18). The short-dashed line shows the corresponding result for $m_{\max }=0$. The long-dashed line shows the result obtained when expansions (2)-(4) are truncated after $m_{\max }=5$ and the contributions from higher $|m|$ are neglected altogether.

Changing the variable $r$ in (32) to $\rho=r / R$ gives the scaled equation

$$
\frac{d^{2} u_{0}^{\mathrm{inc}}}{d \rho^{2}}+\left[\frac{1 / 4}{\rho^{2}}+\frac{\beta}{R} \frac{X(\rho)}{\rho^{3}}\right] u_{0}^{\mathrm{inc}}(\rho)=0 .
$$

The solution of the scaled equation obeys incoming boundary conditions for $\rho \rightarrow 1$ and behaves asymptotically as

$$
u_{0}^{\text {inc }}(\rho) \stackrel{\rho \rightarrow \infty}{\propto}-\sqrt{\rho} \ln \left(\frac{\rho}{\tilde{\mathfrak{a}}}\right)=-\sqrt{\frac{r}{R}} \ln \left(\frac{r}{R \tilde{\mathfrak{a}}}\right) .
$$

Since $\beta / R$ is the only parameter in Eq. (35), the "scaled scattering length" $\tilde{a}$ can only depend on this ratio, $\beta / R$. Comparing (29) and (36) reveals that the true complex scattering length $\mathfrak{a}$ strictly obeys the following scaling behavior:

$$
\mathfrak{a}=R \tilde{\mathfrak{a}}\left(\frac{\beta}{R}\right) .
$$

The modulus and phase of the dimensionless scaled scattering length $\tilde{\mathfrak{a}}$ are shown as functions of $\beta / R$ in Figs. 5 and 6 , respectively. As shown in Fig. 5, $|\tilde{\mathfrak{a}}|$ tends to unity from 


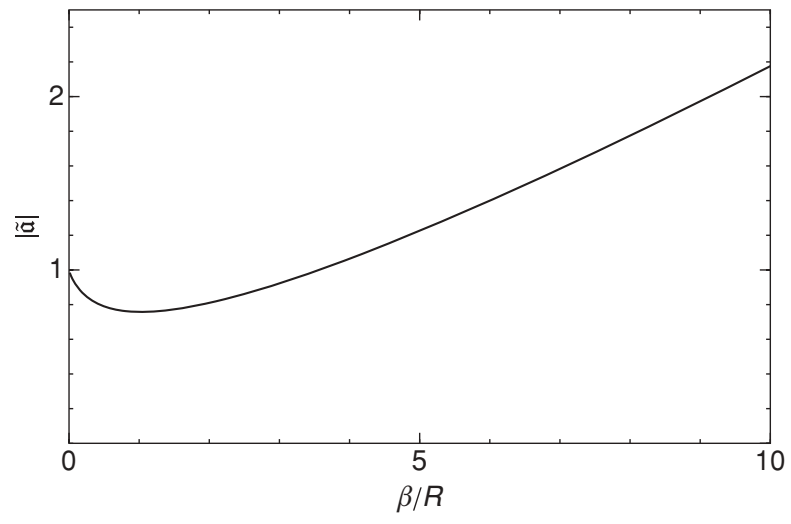

FIG. 5. Modulus $|\tilde{\mathfrak{a}}|$ of the scaled scattering length as a function of $\beta / R$.

below for $\beta / R \rightarrow 0$ and grows (almost) linearly for large $\beta / R$. Note that $|\tilde{\mathfrak{a}}|$ has a minimum value $|\tilde{\mathfrak{a}}|_{\min }=0.7579 \ldots$ for $\beta / R=1.029 \ldots$ The phase $\arg (\tilde{\mathfrak{a}})$ of the scattering length (Fig. 6) varies monotonically from 0 in the thick-wire limit $\beta / R \rightarrow 0$ to $-\pi$ in the thin-wire limit $\beta / R \rightarrow \infty$.

For very thick wires, $R \gg \beta$, the potential term in (35) becomes very small, so that the equation almost corresponds to the free-particle case, except for $\rho \rightarrow 1$, where the behavior (9) of $X(\rho)$ guarantees semiclassical behavior and the possibility of defining incoming boundary conditions. In the near-surface regime, the potential term in $(35)$ becomes $\beta /\left[4 R(\rho-1)^{3}\right]$ and it is dominant as long as $(\rho-1)^{3} \ll \beta / R[\beta / R \ll 1]$. The appropriate wave function obeying incoming boundary conditions for $\rho \rightarrow 1$ is the Hankel function [9]

$$
u_{0}^{\text {inc }}(\rho) \stackrel{\rho \rightarrow 1}{\propto} \frac{2}{y} H_{1}^{(1)}(y), \quad y=\sqrt{\frac{\beta / R}{\rho-1}} .
$$

For sufficiently small values of $\beta / R$, the condition $(\rho-1)^{3} \ll \beta / R$ remains fulfilled even for quite large values of $(\rho-1) R / \beta$, where the small-argument expansion of the Hankel function in (38) is appropriate. Matching to the free-wave behavior for large $\rho$ yields

$$
|\tilde{\mathfrak{a}}| \stackrel{\beta / R \rightarrow 0}{\sim} 1+O\left(\frac{\beta}{R}\right), \quad \arg (\tilde{\mathfrak{a}}) \stackrel{\beta / R \rightarrow 0}{\sim}-\pi \frac{\beta}{4 R} .
$$

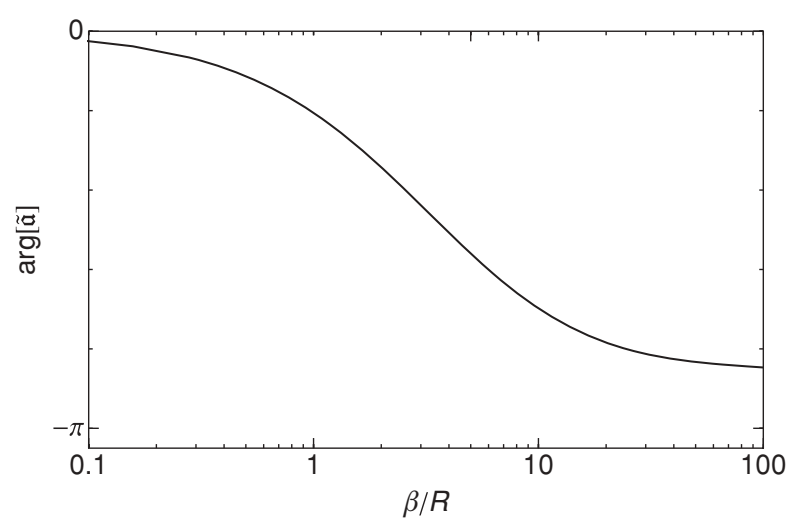

FIG. 6. Phase $\arg (\tilde{\mathfrak{a}})$ of the scaled scattering length as a function of $\beta / R$.

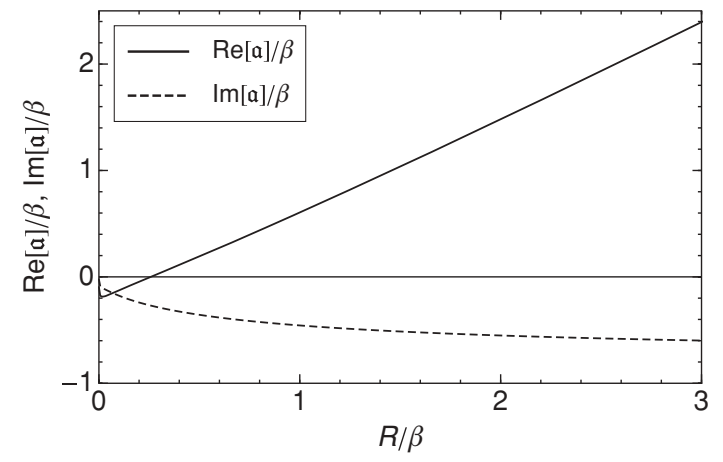

FIG. 7. Real and imaginary parts of the true scattering length as functions of $R / \beta$

The behavior of the true scattering length in the large- $R$ limit is thus

$$
\mathfrak{a}^{R / \beta \rightarrow \infty}[R+O(\beta)] e^{-i \pi \beta /(4 R)},
$$

or, equivalently,

$$
\operatorname{Re}(\mathfrak{a}) \stackrel{R / \beta \rightarrow \infty}{\sim} R+O(\beta), \quad \operatorname{Im}(\mathfrak{a}) \stackrel{R / \beta \rightarrow \infty}{\sim}-\frac{\pi}{4} \beta .
$$

Real and imaginary parts of $\mathfrak{a}$ (in units of $\beta$ ) are plotted as functions of $R / \beta$ in Fig. 7. $\operatorname{Im}(\mathfrak{a})$ falls monotonically from small negative values to $-\frac{\pi}{4} \beta$. The $\operatorname{sign}$ of $\operatorname{Re}(\mathfrak{a})$ changes at $R / \beta \approx 0.25(\beta / R \approx 4)$, where $\arg (\mathfrak{a})$ [which is the same as $\arg (\tilde{\mathfrak{a}})]$ passes through $-\frac{\pi}{2}$ (see Fig. 6). The almost-linear increase in $\operatorname{Re}(\mathfrak{a})$ is more clearly visible in Fig. 8, which covers a larger range of radii.

Note that the imaginary part of the scattering length in the thick-wire limit, namely, $-\frac{\pi}{4} \beta$, is the value one would expect for the imaginary part of the scattering length for a homogeneous attractive potential $-C_{3} / r^{3}$ in one-dimensional scattering or for $s$ waves in the three-dimensional case, when the strength parameter is $C_{3}=\hbar^{2} \beta_{3} /(2 M)$ with $\beta_{3}=\frac{1}{4} \beta$ [22]. This is precisely the situation encountered close to the surface according to (10). So, although the definition of the scattering length is very different from the two-dimensional case, in fact the real part of the scattering length does not exist for $1 / r^{3}$ potentials in conventional one- or three-dimensional scattering, the imaginary part of the conventional scattering length derived from the near-surface potential agrees with the imaginary part of the two-dimensional scattering length in the thick-wire limit.

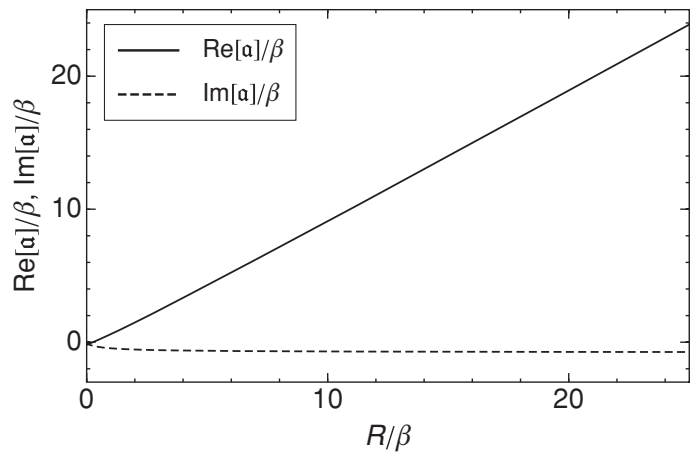

FIG. 8. Real and imaginary parts of the true scattering length as functions of $R / \beta$ for large $R / \beta$. 


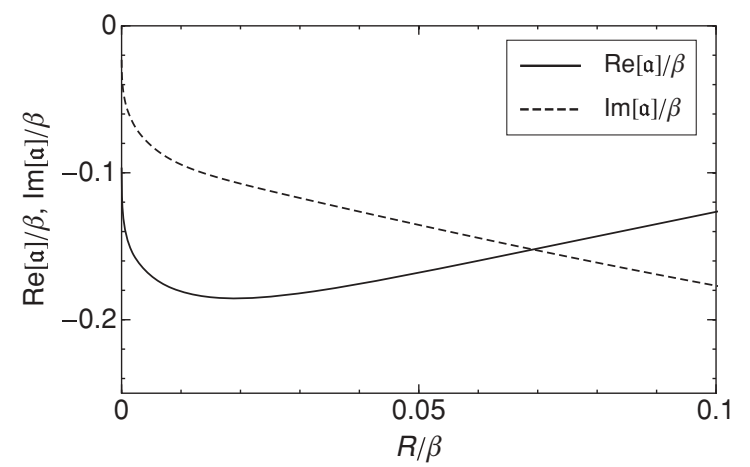

FIG. 9. Real and imaginary parts of the true scattering length as functions of $R / \beta$ for small $R / \beta$.

For very thin wires, $R \ll \beta$, the potential term in (35) becomes very large. For a homogeneous attractive potential $-C_{\alpha} / r^{\alpha}$, the scattering length is proportional to $C_{\alpha}^{1 /(\alpha-2)}$, that is, proportional to the coefficient $C_{\alpha}$ when $\alpha=3$ [12]. The potential term $(\beta / R) X(\rho) / \rho^{3}$ in (35) is close to a $-1 / \rho^{3}$ potential, and its asymptotic falloff is faster than $1 / \rho^{3}$ only by a reciprocal logarithm. We might expect the scaled scattering length to increase almost, but not quite, linearly with the coefficient $\beta / R$ near the thin-wire limit. A linear increase in $\tilde{\mathfrak{a}}$ with $\beta / R$ would imply that the true scattering length $\mathfrak{a}$ tends to a constant in the thin-wire limit. An increase marginally short of linear behavior would mean that $\mathfrak{a}$ tends to 0 very slowly for $R / \beta \rightarrow 0$. This is confirmed in Fig. 9, which shows the real and imaginary parts of the true scattering length $\mathfrak{a}$ for small values of $R / \beta$. Both tend to 0 very slowly. Numerical tests indicate that $\operatorname{Re}(\mathfrak{a})$ tends to 0 marginally slower than $[\ln (R / \beta)]^{-1}$, whereas $\operatorname{Im}(\mathfrak{a})$ tends to 0 marginally faster than $[\ln (R / \beta)]^{-1}$ for $R / \beta \rightarrow 0$.

The modulus $|\mathfrak{a}|$ of the scattering length is a measure of the range of the interaction in the two-dimensional scattering problem, and $s$ waves dominate the cross section when $k|\mathfrak{a}|<1$. When the wire radius is in the nanometer range, the parameters of the nonretarded atom-wire potential are close to the thin-wire limit for realistic atoms; see the discussion in connection with Table I. According to Fig. 9, $|\mathfrak{a}|$ is no larger than about $0.2 \beta$ in this regime, so we can expect the cross section to be accurately given by the near-threshold formula (31) for $k|\mathfrak{a}| \lesssim 0.2 k \beta<1$. Temperatures corresponding to this bound range from the millikelvin scale for hydrogen, to several nanokelvins for lithium, to $<1 \mathrm{nK}$ for the heavier alkalis. Above this bound, the contributions from higher partial waves will make non-negligible contributions to the cross section. Below this bound, $s$ waves are dominant, and for $k \rightarrow 0$, the scattering cross section diverges as $1 / k$ according to (31). The absorption cross section is of the same order of magnitude and diverges in the same way.

\section{CONCLUSION}

The low-energy scattering of a polarizable atom (or molecule) by a conducting cylindrical wire is a fundamental process in the realm of low-temperature physics involving nanotubes. Two features make this seemingly simple problem interesting and different from conventional scattering problems: (i) the nontrivial nature of the atom-wire potential and (ii) the translational symmetry along the axis of the wire, which makes the system effectively two-dimensional.

We have presented a first calculation of atom-wire scattering based on the exact nonretarded potential recently given in Ref. [6]. Generalizing an ansatz proposed in [6] we have formulated a hierarchy of approximations which allow the numerical determination of the nonretarded atom-wire potential to any required accuracy as economically as possible. An essential feature of our approximations to the exact potential is that they reproduce the near-surface behavior (10), where the potential diverges as the inverse cube of the distance between atom and surface.

Assuming incoming boundary conditions (i.e., total absorption) near the surface of the wire we calculated the complex $s$-wave scattering length $\mathfrak{a}$, which dominantly determines the scattering process at near-threshold energies. To obtain accurate values for the scattering length, it is essential to use a potential which accurately reproduces the singular near-surface behavior (10). Approximate potentials with the correct asymptotic behavior up to quite high orders of $1 / r$ still give poor results for the scattering length if they fail to reproduce the near-surface behavior.

The scattering length strictly obeys the scaling law (37), i.e., $\mathfrak{a} / R$ depends only on the ratio of $R$ and a length $\beta$ characteristic of the polarization properties of the (spherical) atom. For typical atoms, these characteristic lengths range from a few thousand to several million atomic units (see Table I).

In the thick-wire limit, the real part of $\mathfrak{a}$ approaches $R+O(\beta)$, while the imaginary part converges to $-\frac{\pi}{4} \beta$. In the thin-wire limit both real and imaginary parts of $\mathfrak{a}$ tend to 0 very slowly.

\section{ACKNOWLEDGMENTS}

This work was supported by the Deutsche Forschungsgemeinschaft, Az.: FR 591/13-2.
[1] R. H. Baughman, A. A. Zakhidov, and W. A. de Heer, Science 297, 787 (2002).

[2] I. R. Lapidus, Am. J. Phys. 50, 45 (1982).

[3] B. J. Verhaar, J. P. H. W. van der Eijinde, M. A. Voermans, and M. M. J. Schaffrath, J. Phys. A 17, 595 (1984).

[4] A. K. Adhikari, Am. J. Phys. 54, 362 (1986).

[5] G. L. Klimchitskaya, U. Mohideen, and V. M. Mostepanenko, Rev. Mod. Phys. 81, 1827 (2009).
[6] C. Eberlein and R. Zietal, Phys. Rev. A 75, 032516 (2007)

[7] Yu. S. Barash and A. A. Kyasov, Sov. Phys. JETP 68, 39 (1989).

[8] C. Eberlein and R. Zietal, Phys. Rev. A 80, 012504 (2009).

[9] M. A. Abramowitz and I. A. Stegun, eds., Handbook of Mathematical Functions (Dover, New York, 1972), Chap. 9. 
[10] G. Barton, J. Phys. B 7, 2134 (1974).

[11] S. Haroche, in Fundamental Systems in Quantum Optics, Les Houches Summer School, Session LIII, edited by J. Dalibard, J.-M. Raimond, and J. Zinn-Justin (North-Holland, Amsterdam, 1992), Course 13, p. 767.

[12] F. Arnecke, H. Friedrich, and P. Raab, Phys. Rev. A 78, 052711 (2008).

[13] N. N. Khuri, A. Martin, J. M. Richard, and T. T. Wu, J. Math. Phys. 50, 072105 (2009).

[14] C. Ticknor, Phys. Rev. A 80, 052702 (2009); 81, 042708 (2010).

[15] F. Arnecke, H. Friedrich, and J. Madroñero, Phys. Rev. A 75, 042903 (2007).
[16] H. A. Bethe and E. E. Salpeter, in Handbuch der Physik, edited by S. Flügge (Springer-Verlag, Berlin, 1957), Vol. XXXV, pp. 88-436.

[17] C. L. Pekeris, Phys. Rev. 115, 1216 (1959).

[18] Z.-C. Yan and J. F. Babb, Phys. Rev. A 58, 1247 (1998).

[19] Z.-C. Yan, A. Dalgarno, and J. F. Babb, Phys. Rev. A 55, 2882 (1997).

[20] P. Kharchenko, J. F. Babb, and A. Dalgarno, Phys. Rev. A 55, 3566 (1997).

[21] A. Derevianko, W. R. Johnson, M. S. Safronova, and J. F. Babb, Phys. Rev. Lett. 82, 3589 (1999).

[22] F. Arnecke, H. Friedrich, and J. Madroñero, Phys. Rev. A 74, 062702 (2006). 